\title{
PCSK9 inhibition and the global diabetes epidemic
}

\author{
Luca Mascitelli ${ }^{1}$ Mark R. Goldstein ${ }^{2}$
}

Received: 12 January 2017 / Accepted: 13 January 2017 / Published online: 29 January 2017

(C) Springer-Verlag Berlin Heidelberg 2017

Keywords Cardiovascular disease $\cdot$ Diabetes risk . Epidemiology $\cdot$ Monoclonal antibody $\cdot$ PCSK9

\section{Abbreviation \\ PCSK9 Proprotein convertase subtilisin/kexin type 9}

To the Editor: Preiss and Mafham [1] analysed the potential efficacy and safety of proprotein convertase subtilisin/ kexin type 9 (PCSK9) inhibitors in reducing major cardiovascular events in clinical practice. However, the diabetogenic effect of PCSK9 inhibition was downplayed by the authors.

Genetically determined loss of PCSK9 function has been shown to increase risk of type 2 diabetes and related variables. In particular, a significant and directionally consistent association between PCSK9 variants and clinical phenotypes has been identified: for each $1 \mathrm{mmol} / 1$ reduction in PCSK9-determined LDL-cholesterol, there was an associated $1.03 \mathrm{~kg}$ increase in body weight, a 0.006 increase in waist-to-hip ratio, a $0.09 \mathrm{mmol} / \mathrm{l}$ increase in fasting glucose, and a significant OR $(95 \% \mathrm{CI})$ of 1.29 $(1.11,1.50)$ for type 2 diabetes [2]. Furthermore, it was recently found that variants in PCSK9 had the same effects

Luca Mascitelli

lumasci@libero.it

1 Comando Brigata Alpina "Julia"/Multinational Land Force, Medical Service, 8 Via S. Agostino, Udine 33100, Italy

2 NCH Physician Group, Naples, FL, USA as variants in the gene encoding 3-hydroxy-3-methylglutaryl-coenzyme A (HMG-CoA) reductase on reducing the risk of cardiovascular events and increasing diabetes risk per unit decrease in LDL-cholesterol level [3]. Unfortunately, the effect of these genetic variants on total mortality is unknown.

Mounting evidence has established that risk of death varies significantly among individuals with familial hypercholesterolaemia [4]. Moreover, the excess mortality from this disorder has varied over time, and in the 19th century, when infectious disease was prevalent, total mortality was lower than in the general population, thus suggesting that even in monogenic disorders, other genes and the environment can be important [5].

Notably, the rate of decline in cardiovascular events has decelerated substantially in recent years [6]. A significant concern is the possibility that cardiovascular disease mortality rates may stop decreasing and may even increase in future, in part owing to the increasing prevalence of obesity and diabetes at epidemic proportions. In this environment, genetic variants that further increase diabetes risk may, in fact, no longer be protective.

In trials of PCSK9 inhibitor drugs, and specifically in the context of the real world global diabetes epidemic, physicians should carefully assess the possible diabetogenic action of PCSK9 inhibitor treatment. 
Funding This work received no specific funding.

Duality of interest The authors declare that there is no duality of interest associated with this manuscript.

Contribution statement Both authors were responsible for drafting the article and revising it critically for important intellectual content. Both authors approved the version to be published.

\section{References}

1. Preiss D, Mafham M (2016) PCSK9 inhibition: the dawn of a new age in cholesterol lowering? Diabetologia. doi:10.1007/s00125-016-4178-y

2. Schmidt AF, Swerdlow DI, Holmes MV et al (2016) PCSK9 genetic variants and risk of type 2 diabetes: a mendelian randomisation study. Lancet Diabetes Endocrinol. doi:10.1016/S2213-8587(16) 30396-5

3. Ference BA, Robinson JG, Brook RD et al (2016) Variation in PCSK9 and HMGCR and risk of cardiovascular disease and diabetes. N Engl J Med 375:2144-2153

4. Williams RR, Hasstedt SJ, Wilson DE et al (1986) Evidence that men with familial hypercholesterolemia can avoid early coronary death. An analysis of 77 gene carriers in four Utah pedigrees. JAMA 255: 219-224

5. Sijbrands EJ, Westendorp RG, Defesche JC, de Meier PH, Smelt AH, Kastelein JJ (2001) Mortality over two centuries in large pedigree with familial hypercholesterolaemia: family tree mortality study. BMJ 322:1019-1023

6. Sidney S, Quesenberry CP Jr, Jaffe MG et al (2016) Recent trends in cardiovascular mortality in the United States and public health goals. JAMA Cardiol 1:594-599 